\title{
Inguinal Lymphadenectomy Assessment Associated with Penile Carcinoma Undergone New Strategies for Nodal Staging
}

\author{
${ }^{1}$ Department of Surgery, Hospital Sierrallana, Cantabria, Spain \\ ${ }^{2}$ Department of Radiology, Hospital Sierrallana, Cantabria, Spain \\ ${ }^{3}$ Department of Urology, Hospital Sierrallana, Cantabria, Spain \\ ${ }^{4}$ Universidad Autónoma, Madrid, Spain \\ Email: *vovejerohcas@msn.com
}

Victor J. Ovejero-Gomez ${ }^{1 *}$, L. Martin-Cuesta ${ }^{2}$, F. Martinez-Bretones ${ }^{3}$, T. Gallego-Bellido', L. A. Asensio-Lahoz ${ }^{3}$, J. Villalba-Torre ${ }^{1}$, A. Ingelmo-Setien'1, J. M. Bajo-Arenas ${ }^{4}$

Received 18 April 2014; revised 17 May 2014; accepted 15 June 2014

Copyright @ 2014 by authors and Scientific Research Publishing Inc.

This work is licensed under the Creative Commons Attribution International License (CC BY).

http://creativecommons.org/licenses/by/4.0/

c) (i) Open Access

\section{Abstract}

Penile carcinoma is an uncommon urological tumour and provides an opportunity for curation on early stage of the disease. Nodal metastases are one of the most important prognostic factors for survival although detection of inguinal adenopathies could be related with an inflammatory or infectious etiology. A suspicion of bilateral metastasic nodal involvement should be taken with caution. Radical inguinal lymphadenectomy has been associated to a great deal of complications. Several anatomical studies have reported the true lymphatic drainage pathways in order to reduce the area of groin dissection. Nonetheless, a prophylactic modified inguinal lymphadenectomy should not be a systematic surgical procedure in all patients due to morbidity and questioned usefulness when there are not nodal metastasis. Classical imaging studies have a limited contribution to the diagnosis of lymph node metastasis. Nowadays, lymph node involvement may be diagnosed both minimally invasive and noninvasive techniques, such as dynamic sentinel lymph node biopsy in intermediate and high risk patients with nonpalpable lymph nodes, and fine needle aspiration biopsy in cases with palpable nodes. Their high effectiveness has facilitated the radical pelvic or inguinal lymphadenectomy that is only performed when there is histological confirmation of nodal involvement. A new video endoscopic technique has been developed at present to reduce postoperative complications although prospective studies are needed to assess outcomes. The appearance of adenopathies after surgical treatment of the primary tumour could be supported at the same guidelines. An inguinal lymphadenectomy should be carried out in selected patients to support a benefit on early stages with an extended survival.

\footnotetext{
${ }^{*}$ Corresponding author.
} 


\section{Keywords}

\section{Carcinoma, Penis, Surgical Treatment, Lymphadenectomy, Nodal Staging}

\section{Introduction}

Penile cancer is an aggressive and rare neoplasia, particularly in developed countries, that originates in the epithelium of the glands and of the inner prepuce. This tumour represents $0.4 \%-0.6 \%$ of all neoplasias [1].

The world's highest prevalence rates are found in India and Brazil. The incidence of this urogenital tumour in the United States and Europe is approximately 1 of 100,000 males per year [2].

It has been associated to human papiloma virus in nearly 50\% cases although any chronic inflammatory process in the coronal sulcus or poor hygiene encourages its appearance. Infant circumcision could be as a reducing risk factor. In fact, one of the lowest incidence is among Jewish men born in Israel [3].

Peak incidence is between the sixth and seventh decade of life. The earlier the diagnosis, the better the prognosis; considered curable until Stage II of the American Joint Committee on Cancer (AJCC) classification.

Cure depends on control of the primary tumour and regional nodes. The most common sites of metastasis are the superficial and deeper nodes of the inguinal and iliac region. Lymphatic invasion occurs in $50 \%$ of the patients with enlarged inguinal adenopathies and $10 \%-15 \%$ who present with nonpalpable lymph nodes, even in small tumours [4]. Distant dissemination is usually late.

This cancer may be found in a locoregional stage for a long time, which explains that inguinal lymphadenectomy can be considered curative in many patients [5].

Nonetheless, the presence of inguinal nodes in this carcinoma is common as rich inflammatory response to bacterial flora of the region or local infection, especially after an isolated resection of the primary tumour.

The persistence of these lymph nodes after three weeks of removal of the primary lesion and proper antibiotic coverage should advise performing an inguinal dissection. In this regard, the European Urological Association recommends lymphadenectomy for all patients with clinically palpable inguinal lymph nodes [3].

Nowadays, both minimally invasive and noninvasive staging strategies have been developed for the identification of patients with lymph node involvement. The contribution of these new strategies for nodal staging could reduce the magnitude of complications caused by the inguinal dissection.

We present a case report of a patient diagnosed with squamous cell carcinoma of the penis and some risk factors of lymph node metastasis according to the European Urological Association's guidelines who evolved superficial and deep lymph node metastasis in early follow-up and underwent bilateral radical inguinal lymphadenectomy. We evaluate this surgical procedure for this type of cancer in conjunction with other diagnostic techniques for nodal infiltration.

\section{Case Report}

A 77-year-old man with past medical history of transient ischemic attack, myocardial infarction and atrial fibrillation consulted on an outpatient basis by a preputial tumour with glans involvement for the past month.

No palpable inguinal lymph nodes were found on physical examination.

The pacient underwent subtotal penectomy and diagnosed with ulcerated squamous cell carcinoma with invading the subepithelial connective tissue, moderately differentiated, 2.5 centimeters in maximum diameter, respecting the oncological resection borders.

A few small bilateral superficial inguinal adenopathies were found at 6 months follow-up. They were treated with antibiotic therapy without any success. In the clinical course, these lymph nodes grew in size and number, as confirmed with abdominopelvic computed tomography (CT) scan (Figure 1). A fine needle aspiration biopsy was performed to confirm recurrence. Other imaging studies excluded the possibility of distant metastasis.

We performed a bilateral superficial and deep inguinal lymphadenectomy (Figure 2), sending intraoperative lymph samples from both sides which were negative for malignancy and iliac dissection was dismissed.

He suffered from a postoperative well-tolerated right lymphorrhagia which was treated with a conservative management.

Histopathological diagnosis was reported moderately differentiated squamous cell carcinoma in five right and 


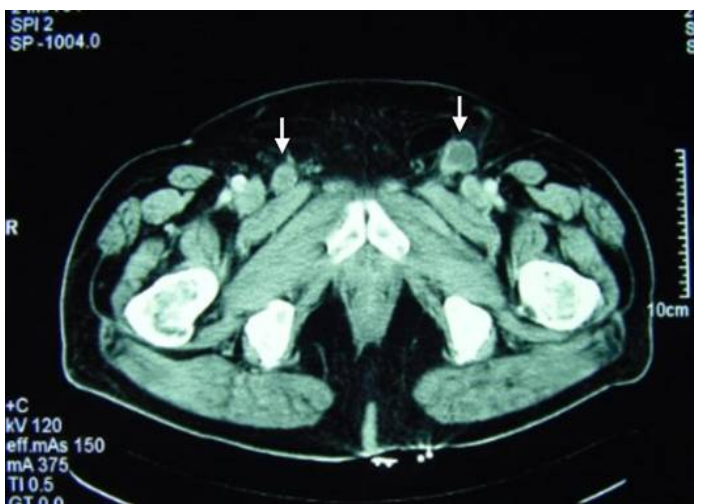

(a)

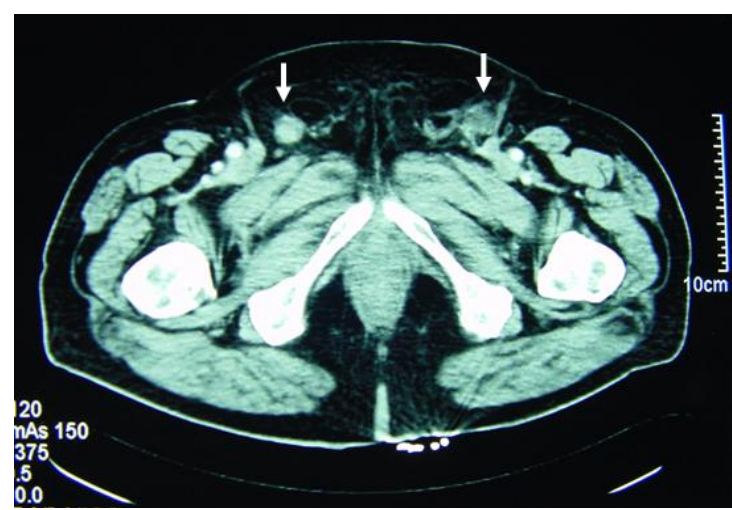

(b)

Figure 1. Imaging study using computed tomography scan for the diagnosis of lymph node metastases. Appearance of pathological lymph nodes in both inguinofemoral regions (arrows), some of them with focal necrosis (a) or involvement of the fatty tissue around the lymph nodes (b).

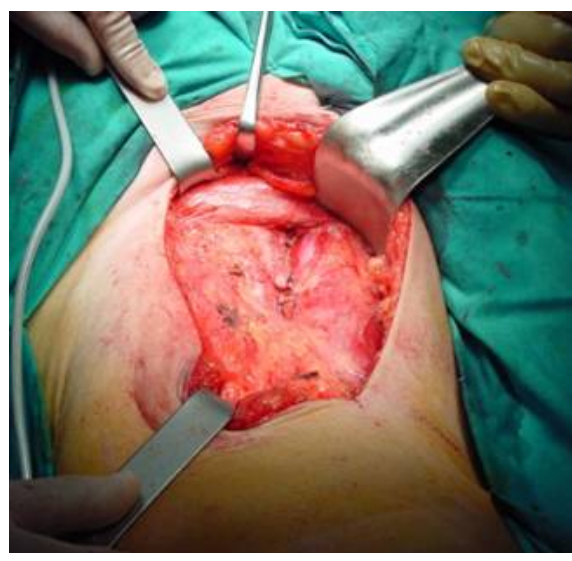

(a)

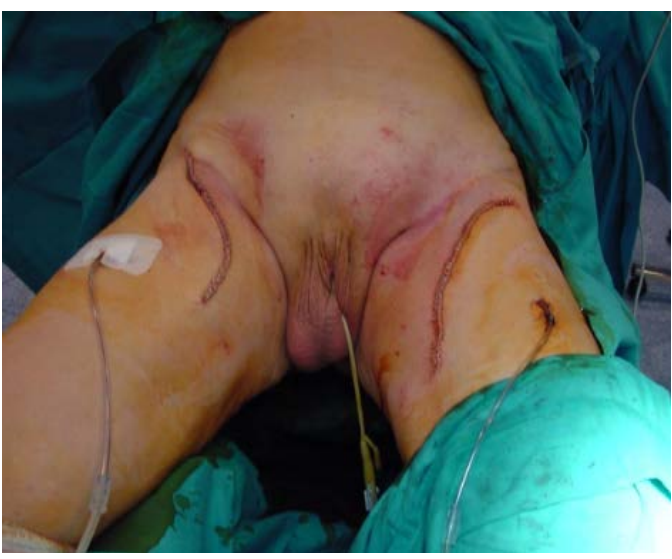

(b)

Figure 2. Operative field after cutaneous flaps have been developed: (a) Inguinocrural region showing the anatomical limits of the nodal dissection after lymph nodes, saphena magna vein and the subcutaneous tissue were removed. The vascular ligation at the arch of the saphenous vein can be observed; (b) Aspect of the surgical area in both inguinocrural regions after lymphadenectomy highlighting the shape of the cutaneous incision and use of closed suction dranaiges.

four left lymphadenopathies, presenting extracapsular involvement and infiltration of perinodal fatty tissue.

This penile carcinoma was staged as $\mathrm{G}_{2} \mathrm{pT}_{1 \mathrm{a}} \mathrm{pN}_{3} \mathrm{M}_{0}$ (Stage IV) according to $7^{\text {th }}$ edition of the AJCC Cancer Staging Manual. An iliac lymphadenectomy and neoadjuvant chemotherapy was recommended but our patient refused.

Finally, he only underwent radiotherapy in the surgical areas and a cutaneous ulceration appeared as complication to radiation therapy.

We performed a follow-up by physical examination and CT scan. Our patient was disease free for nine months. Then, he was attended complaining of dysnea and a pulmonary X-ray showed metastasis in both lungs.

He accepted a palliative treatment and died of respiratory failure a month later.

\section{Discussion}

Inguinal lymph node metastases are common in penile carcinoma because these nodes are responsible for primary drainage of genitalia whereas secondary drainage is to the pelvic nodes.

Clinical and radiological assessment of the inguinal lymph nodes is a controversial issue related to both diagnosis and follow-up of this carcinoma. Nodal metastasis represents one of the most important prognostic factor of the disease [6], with $28 \%$ - $64 \%$ of the cases detected when diagnosis is made, although a quarter of patients 
could have metastasic adenopathies without palpable lymph nodes [7].

In fact, there is a high incidence of microscopic lymph node metastasis, even in early stages, not demonstrated at time of diagnosis, that will have an impact in disease prognosis.

From an anatomical point of view, inguinal nodes can be divided into a superficial, just below subcutaneous, and deeper group around the femoral vein. Nonetheless, Daseler [8] functionally divided this region into four quadrants and a central zone at the junction of the femoral and saphenous veins. Later, several lymphangiographic studies of the penis [9] reported no direct drainage to the lowest quadrants and pelvic region. These findings suggested that a modified inguinal lymphadenectomy might be feasible to reduce the area of groin dissection in order to minimize morbidity associated to radical lymphadenectomy and preserve the therapeutic benefit.

The most common complications in the radical procedure are seroma, lymphocele, skin edge necrosis, wound infection, lymphedema, flap necrosis, scrotal edema and deep venous thrombosis [10]. A high rate of them has been described in patients with a high body mass index, sartorius muscle transposition or bilateral dissection.

These factors could be considered strongly associated with the risk of postoperative complications [11].

Neither high morbidity in radical lymphadenectomy, reported between $30 \%$ and $50 \%$ of cases [12], nor its real unknown effect in survival rates as prophylactic measure should justify this systematic surgical porcedure without a high level of suspicion of lymph node involvement.

Nowadays, extent of lymph node dissection should be adapted to clinical stage. Lymphadenectomy should not be indicated in low risk patients ( $\mathrm{pTis}, \mathrm{pTaG}_{1-2}, \mathrm{pT}_{1} \mathrm{G}_{1}$ ) without palpable lymph nodes. On the contrary, it could be indicated in all patients with clinically palpable inguinal lymph nodes at diagnosis or high $\left(\mathrm{pT}_{2-4}, \mathrm{G}_{3}\right.$ or vascular invasion) and intermediate $\left(\mathrm{T}_{1} \mathrm{G}_{2}\right.$ with vascular invasion or nodular growth pattern) risk of nodal metastasis according to the European Urological Association's guidelines, because of the potential risk of lymph node metastasis [13].

An alternative to this surgical procedure is a dynamic sentinel lymph node biopsy in the presence of nonpalpable nodes and risk factors or fine needle biopsy in cases of palpable adenopathies. Nonetheless, patients with histologically proven nodal metastasis should undergo radical inguinal lymphadenectomy, extended to the pelvis if there are more than two lymph nodes affected, to improve survival [1].

A new procedure, known as video endoscopic inguinal lymphadenectomy (VEIL) [14], has recently been proposed as a safe option to reduce postoperative morbidity in classical lymphadenectomy, both palpable and non-palpable nodes. A few centres around the world have performed this technique and several prospective multicenter studies will be needed to assess its long-term oncological outcome despite its initial success.

Neoadjuvant chemotherapy is recommended in fixed lymph nodes before resection.

Both sentinel node and fine needle biopsy in combination with ultrasound scan may also be of great value in detecting lymph node metastases during follow-up of these patients with high sensitivity and specificity, even in non-palpable disease. Their main advantage is to decrease the open lymphadenectomy-related mobidity with a low false-negative and complications rate of around 5\% [15] [16].

Other classical diagnostic techniques, such as CT scan, magnetic resonance imaging (MRI) or positron emission tomography-CT (PET-CT), are unable to detect micro-metastases and have a poor contribution to the diagnosis of lymph node recurrence [17], except in cases with high clinical suspicion.

A delayed lymphadenectomy, involvement of more than one lymph node or deep lymph group, high grade and extranodal extension are associated with a high risk of nodal recurrence for the postoperative follow-up and worsen prognosis of the disease.

In any case, the presence of enlarged palpable inguinal adenopathies at diagnosis or during the postoperative follow-up should be taken with caution as there is $30 \%$ false-positive nodes [18] due to locoregional inflammatory or infectious processes and antibiotic therapy has not proven effectiveness in reducing the lymph node swelling.

This relative contribution of antibiotics has justified another operation until recently to rule out nodal recurrence despite the absence of risk factors in the resection of the primary tumour. A sensitivity and specificity more than 90\% in cytology by fine needle aspiration could help determine the actual need for radical inguinal lymphadenectomy [19] or a clinical observation when antibiotic treatment is completed.

Histological confirmation of a unilateral palpable nodal metastasis at diagnosis of disease does not rule out involvement of both groins, present in 50\% of cases due to lymphatic drainage crossed penis under physiological conditions [20]. In these patients, it could be recommended both superficial and deep lymphadenectomy of 
the affected side but only superficial in contralateral side, considering the usual existence of a wide distribution of nodes in the superficial region compared to the deep lymph nodes.

The other way around, the absence of palpable lymph nodes in the contralateral side after resection of the primary tumour could exclude its involvement because it would be expected a lymphatic growth at the same speed in both sides. In this context, contralateral lymphadenectomy will exclude unless malignancy was suspected in some imaging study.

The development of new therapeutic approaches such as video endoscopic inguinal lymphadenectomy, could reduce overall postoperative morbidity in these cases, but the actual worldwide experience is still very limited.

In clinical practice, there are many doubts about the usefulness of the ilioinguinal dissection at the same surgical time because of their high risk of complications. Most authors [1] only advise a pelvic lymphadenectomy when there is a histological confirmation of two or more positive lymph nodes for malignancy in radical inguinal dissection.

Pelvis lymph node involvement is used to being more useful for staging than for treatment of the disease because of Stage IV is only amenable to a palliative therapy in order to relieve symptoms or improve quality of life, without changing the natural history of the cancer.

The evolution of the disease to early nodal recurrence suggests that our patient could have been a good candidate for a modified inguinal lymphadenectomy or sentinel node biopsy at diagnosis. The existence of risk factors such as tumor size, moderate cell differentiation or the possibility of micrometastases support this therapeutic approach.

In conclusion, prophilactic inguinal lymphadenectomy is not always indicated for penile cancer because it may be associated with significant morbidity. All patients without palpable lymphadenopathies at diagnosis should therefore be considered for a modified node dissection or sentinel node biopsy in experienced centres, except those with low risk of recurrence and good compliance.

Palpable inguinal nodes should not be considered as metastasic disease if there is no histological confirmation. Positive fine needle biopsy can support a radical inguinal lymphadenectomy due to the limited diagnostic value of the current imaging studies to detect nodal metastasis in this cancer.

\section{References}

[1] Protzel, C., Alcaraz, A., Horenblas, S., Pizzocaro, G., Zlotta, A. and Hakenberg, O.W. (2009) Lymphadenectomy in the Surgical Mamagement of Penile Cancer. European Urology, 55, 1075-1088. http://dx.doi.org/10.1016/j.eururo.2009.02.021

[2] Solsona, E., Algaba, F., Horenblas, S., Pizzocaro, G. and Windahl, T. (2004) EAU Guidelines on Penile Cancer. European Urology, 46, 1-8. http://dx.doi.org/10.1016/j.eururo.2004.03.007

[3] Pizzocaro, F., Algaba, F., Horenblas, S., Solsona, E., Tana, S., Van Der Piel, H., et al. (2010) EAU Penile Cancer Guidelines 2009. European Urology, 57, 1002-1012. http://dx.doi.org/10.1016/j.eururo.2010.01.039

[4] Horenblas, S. (2001) Lymphadenectomy for Squamous Cell Carcinoma of the Penis. Part 1: Diagnosis of Lymph Node Metastasis. BJU International, 88, 467-72. http://dx.doi.org/10.1046/j.1464-410X.2001.00380.x

[5] Hegarty, P.K., Rees, R.W., Borley, N.C., Ralph, D.J. and Minhas, S. (2008) Contemporary Management of Penile Cancer. BJU International, 102, 928-932. http://dx.doi.org/10.1111/j.1464-410X.2008.07773.X

[6] Burt, L.M., Shrieve, D.C. and Tward, J.D. (2014) Stage Presentation, Care Patterns and Treatment Outcomes for Squamous Cell Carcinoma of the Penis. International Journal of Radiation Oncology*Biology*Physics, 88, 94-100. http://dx.doi.org/10.1016/j.ijrobp.2013.08.013

[7] Shuck-Bello, C.E., Morales-Montor, J.G., Camarena-Reynoso, H.R., Leos-Acosta, C.A., Castellano-Orozco, M., Parraguirre-Sara, C., et al. (2010) Linfadenectomía pélvica bilateral laparoscópica e inguinal en cáncer sarcomatoide de pene. Revista Mexicana de Urología, 70, 319-324.

[8] Daseler, E.H., Anson, B.J. and Reimann, A.F. (1948) Radical Excision of Inguinal and Iliac Lymph Glands: A Study Based upon 450 Anatomical Dissections and upon Supportive Clinical Observations. Surgery, Gynecology Obstetrics, 87, 679-694.

[9] Cabanas, R.M. (1977) An Apporoach for Treatment of Penile Carcinoma. Cancer, 39, 456-466. http://dx.doi.org/10.1002/1097-0142(197702)39:2<456::AID-CNCR2820390214>3.0.CO;2-I

[10] Koifman, L., Hampl, D., Koifman, N., Vides, A.J. and Ornellas, A.A. (2013) Radical Open Inguinal Lymphadenectomy for Penile Carcinoma: Surgical Technique, Early Complications and Late Outcomes. Journal of Urology, 190, 2086-2092. http://dx.doi.org/10.1016/j.juro.2013.06.016 
[11] Stuiver, M.M., Djajadiningrat, R.S., Graafland, N.M., Vincent, A.D., Lucas, C. and Horenblas, S. (2013) Early Wound Complications after Inguinal Lymphadenectomy in Penile Cancer: A Historical Cohort Study and Risk-Factor Analysis. European Urology, 64, 486-492. http://dx.doi.org/10.1016/j.eururo.2013.02.037

[12] Ornellas, A.A., Seixas, A.L., Marota, A., Wisnescky, A., Campos, F. and de Moraes, J.R. (1994) Surgical Treatment of Invasive Squamous Cell Carcinoma of the Penis: Retrospective Analysis of 350 Cases. Journal of Urology, 151, 1244-1249.

[13] Johnson, T.V., Hsiao, W., Delman, K.A., Jani, A.B., Brawley, O.W. and Master, V.A. (2010) Extensive Inguinal Lymphadenectomy Improves Overall 5-Year Survival in Penile Cancer Patients. Cancer, 15, 2960-2966. http://dx.doi.org/10.1002/cncr.25091

[14] Carlos, A.S., Romanelli, P., Nishimoto, R., Montoya, L.M., Juliano, C.A., da Costa Jr., R.M., et al. (2013) Expanded Criteria for Video Endoscopic Inguinal Lymphadenectomy (VEIL) in Penile Cancer: Palpable Lymph Nodes. International Brazilian Journal of Urology, 39, 893-894. http://dx.doi.org/10.1590/S1677-5538.IBJU.2013.06.17

[15] Djajadiningrat, R.S., Teertstra, H.J., van Werkhoven, E., van Boven, H.H. and Horenblas, S. (2014) Ultrasound Examination and Fine Needle Aspiration Cytology-Useful for Follow up of the Regional Nodes in Penile Cancer? Journal of Urology, 191, 652-625. http://dx.doi.org/10.1016/j.juro.2013.08.046

[16] Lam, W., Alnajjar, H.M., La-Touche, S., Perry, M., Sharma, D., Corbishley, C., et al. (2013) Dynamic Sentinel Lymph Node Biopsy in Patients with Invasive Squamous Cell Carcinoma of the Penis: A Prospective Study of the Long-Term Outcome of 500 Inguinal Basins Assessed at a Single Institution. European Urology, 63, 657-663. http://dx.doi.org/10.1016/j.eururo.2012.10.035

[17] Rosevear, H.M., Williams, H., Collins, M., Lightfoot, A.J., Coleman, T. and Brown, J.A. (2012) Utility of 18F-FDG PET/CT in Identitying Penile Squamous Cell Carcinoma Metastasic Lymph Nodes. Urologic Oncology, 30, 723-726. http://dx.doi.org/10.1016/j.urolonc.2010.09.015

[18] Hegarty, P.K., Kayes, O., Freeman, A., Christopher, N., Ralph, D.J. and Minhas, S. (2006) A Prospective Study of 100 Cases of Penile Cancer Managed According to Ueropean Association of Urology guidelines. BJU International, 98, 526-531. http://dx.doi.org/10.1111/j.1464-410X.2006.06296.x

[19] Saisorn, I., Lawretschuk, N., Leewansangtong, S. and Bolton, D.M. (2006) Fine-Needle Aspiration Cytology Predicts Inguinal Lymph Node Metastasis without Antibiotic Pretreatment in Penile Carcinoma. BJU International, 97, 12251228. http://dx.doi.org/10.1111/j.1464-410X.2006.06159.x

[20] Ekstrom, T. and Edsmyr, F. (1958) Cancer of the Penis, a Clinical Study of 229 Cases. Acta Chirurgica Scandinavica, 115, 25-55. 
Scientific Research Publishing (SCIRP) is one of the largest Open Access journal publishers. It is currently publishing more than 200 open access, online, peer-reviewed journals covering a wide range of academic disciplines. SCIRP serves the worldwide academic communities and contributes to the progress and application of science with its publication.

Other selected journals from SCIRP are listed as below. Submit your manuscript to us via either submit@scirp.org or Online Submission Portal.
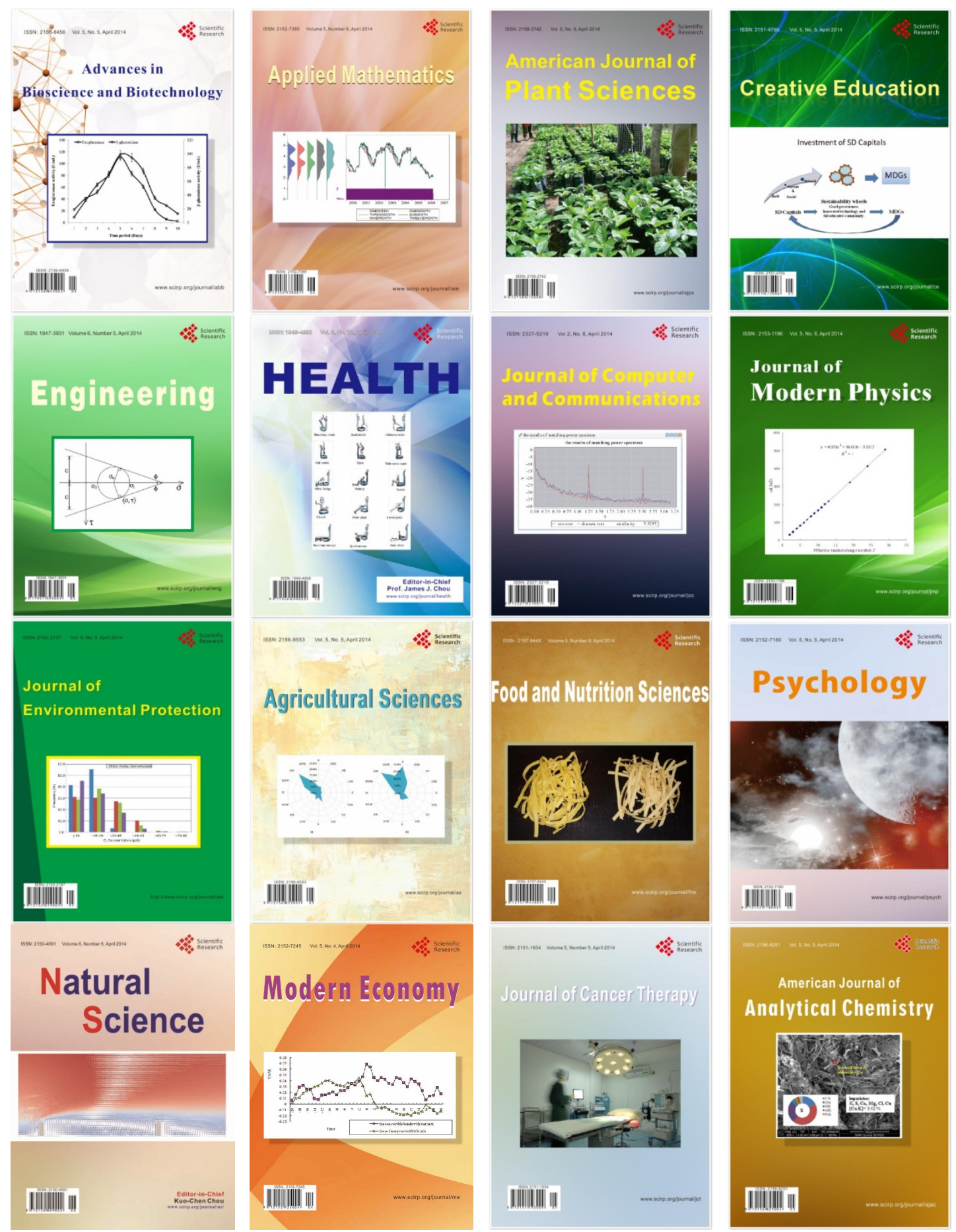